\title{
Noninvasive Dynamic Fluorescence Imaging of Human Melanomas Reveals that Targeted Inhibition of bFGF or FGFR-1 in Melanoma Cells Blocks Tumor Growth by Apoptosis
}

\author{
Marianne Valesky, ${ }^{1}$ Aaron J. Spang, ${ }^{1}$ Gregory W. Fisher, ${ }^{2}$ Daniel L. Farkas, ${ }^{2}$ and Dorothea Becker ${ }^{1}$ \\ ${ }^{1}$ Department of Pathology, University of Pittsburgh, Pittsburgh, PA, USA \\ ${ }^{2}$ Center for Light Microscope Imaging and Biotechnology, Carnegie Mellon University, Pittsburgh, PA, USA \\ Accepted February 4, 2002
}

\begin{abstract}
Background: Two prominent biological features of the advanced stages of human melanoma are their high degree of vascularity and high-level expression of basic fibroblast growth factor (bFGF) and fibroblast growth factor receptor-1 (FGFR-1). Given these characteristics, human melanoma serves as an ideal model to address an important question regarding the efficacy of angiogenesis-based cancer therapy. To induce tumor growth arrest and regression, does it suffice to block expression of bFGF and/or FGFR-1 in only the melanoma cells, or is it essential to inhibit expression of bFGF and/or FGFR-1 in both the melanoma cells and the melanoma cell-interspersing vasculature?

Materials and Methods: Primary and metastatic human melanomas, grown as subcutaneous tumors in nude mice, were injected twice a week with vector constructs containing the human tyrosinase promoter and antisenseoriented human bFGF or FGFR-1 cDNA. On alternating days, the bFGF and FGFR-1 antisense-targeted tumors received injections of cyanine fluorochrome-conjugated antibodies to a human melanoma and mouse blood vessel
\end{abstract}

marker. Noninvasive, dynamic fluorescence imaging was used to document the cellular events that took place inside the tumors as the result of blocking expression of bFGF or FGFR-1 in the melanoma cells.

Results: In vivo, ex vivo, and in vitro fluorescence imaging of the bFGF and FGFR-1 antisense-targeted tumors demonstrated that inhibiting bFGF and FGFR-1 signaling in only the melanoma cells suffices to inhibit tumor growth due to massive induction of melanoma cell apoptosis.

Conclusions: The investigations presented in this study document that inhibiting expression of bFGF or FGFR-1 in only the melanoma cells is as effective in blocking tumor growth as simultaneously inhibiting bFGF or FGFR1 synthesis in the melanoma cells and the melanoma cellinterspersing vasculature. Furthermore, blocking expression of bFGF or FGFR-1 in the melanoma cells did not lead to activation or increased production of another angiogenic molecule, suggesting the absence of a "salvage pathway" that can circumvent or rescue the blockage of bFGF/FGFR-1 in the melanoma cells.

\section{Introduction}

Unlike normal melanocytes, primary melanomas in the vertical growth phase (VGP) and melanomas in the metastatic growth phase (MGP) express high levels of basic fibroblast growth factor (bFGF) and fibroblast growth factor receptor-1 (FGFR-1). Regarding the function of these two genes in advanced stage melanomas, previous in vitro studies revealed that expression of $\mathrm{bFGF} / \mathrm{FGFR}-1$ is essential for the proliferation of VGP and MGP melanoma cells $(1,2,3)$. Furthermore, antisense targeting of bFGF/FGFR-1 in VGP and MGP melanomas, grown as subcutaneous tumors in nude mice, demonstrated inhibition of tumor growth and regression as a result of blocked intratumoral angiogenesis (4). Given these findings, the present study was designed to address two important,

Address correspondence and reprint requests to: Dorothea Becker, PhD, Professor, Department of Pathology, University of Pittsburgh, BST, E1057, 211 Lothrop Street, Pittsburgh, PA 15213, USA. Phone: (412) 624-1047; fax: (412) 624-1048; e-mail: dbecker@pitt.edu. yet unresolved questions with respect to potential angiogenesis-based melanoma therapy. First, in a neoplasm such as melanoma, which produces high levels of bFGF/FGFR-1, does intratumoral angiogenesis constitute a process that involves bFGF/FGFR-1 signaling from the tumor cells to the endothelial cells lining the intratumoral blood vessels, or does it involve bidirectional signaling from the tumor cells to the tumor vasculature and vice versa? Second, what is the biologic mechanism(s) that leads to melanoma growth arrest following targeted inhibition of bFGF/FGFR-1 in only the melanoma cells versus simultaneous inhibition of bFGF/FGFR- 1 in the melanoma cells and the melanoma-interspersing blood vessels?

Given the recent advances in optical imaging combined with the application of cyanine-based fluorochromes that fluoresce in the near-infrared $(5,6)$, it has become possible to visualize, noninvasively and in real time, dynamic changes in living biological systems that cannot be captured by conventional light microscopy (7). Conducting noninvasive, dynamic fluorescence imaging of human melanomas 
injected with human tyrosinase (Tyr), Rous Sarcoma Virus (RSV) promoter-driven bFGF, or FGFR-1 antisense construct and fluorochrome-conjugated antibodies to a human melanoma and mouse blood vessel marker, the findings presented here demonstrate that antisense targeting of bFGF and, likewise, FGFR-1 in only the melanoma cells is as effective in inhibiting tumor growth as blocking expression of bFGF and FGFR-1 simultaneously in the melanoma cells and the melanoma cell-interspersing blood vessels. In addition, the results of this macroscopic and microscopic cyanine fluorochrome-based optical imaging study provides the first evidence that targeting bFGF or FGFR-1 in the melanoma cells induces extensive melanoma cell-specific apoptosisa process the melanoma cells are unable to circumvent by activating or increasing expression of another growth factor/receptor.

\section{Materials and Methods \\ Cell Culture}

Human VGP (WM 98-2; WM 983-A) and MGP (WM 983-B) melanoma cell lines were cultured as previously described $(2,3)$.

\section{Vector Constructs}

A 430-bp human tyrosinase promoter cDNA fragment (8) was subcloned via an Xho I and Xba I restriction site to replace a 670-bp RSV promoter-specific cDNA in pREP7 plasmids (Invitrogen, Carlsbad, CA, USA), containing an antisense-oriented, full-length $1.4-\mathrm{kb}$ human bFGF cDNA and an antisense-oriented, fulllength 3.3-kb FGFR-1 cDNA (4). Sequence analysis ensured correct insertion and orientation of the human tyrosinase promoter in the respective vectors.

\section{Cyanine Fluorochrome Conjugation of Antibodies}

Conjugations of antibodies with $\mathrm{Cy} 3$ or Cy5 were performed with commercially available CyDye Fluorolink antibody labeling kits (Amersham Pharmacia Biotech, Piscataway, NJ, USA). Activation of Cy7 and Cy7 fluorochrome-labeling of antibodies were carried out as previously described (5). Antibodies used in these studies were anti-cow S100 monoclonal antibody (Dako Corporation, Carpenteria, CA, USA), antimouse CD31 monoclonal antibody (PharMingen, San Diego, CA, USA), anti-human bFGF and FGFR-1 monoclonal antibodies (Selective Genetics, San Diego, CA, USA), and anti-human VEGF-D monoclonal antibody (R\&D Systems, Minneapolis, MN, USA).

\section{Noninvasive Dynamic Fluorescence Imaging}

A modified Nikkor (Nikon, Melville, NY, USA) 50mm lens, attached to a C250 Peltier-cooled chargecoupled device (CCCD) camera (Photometrics, Waterloo, Ontario, Canada), was used to visualize and record images of human VGP and MGP melanomas, grown as subcutaneous tumors in nude mice (nu/nu, BALB/c background, Taconic). The tumor-bearing animals were sedated for a short period and placed on a stage mounted below the camera lens. Excitation light was delivered via a split-fiber optics source placed above the animals. Live images of tumors were captured through the skin of the mice with exposure times of $30 \mathrm{sec}$. The filters (Chroma Technology, Brattleboro, VT, USA) were as follows: for Cy3 (excitation $530 \mathrm{~nm}$, emission $590 \mathrm{~nm}$ ), Cy5 (excitation $640 \mathrm{~nm}$, emission $670 \mathrm{~nm}$ ), and Cy7 (excitation $710 \mathrm{~nm}$, emission $810 \mathrm{~nm}$ ). Exposure times and illumination intensities were equalized for intensity-based comparisons.

Noninvasive, live images of tumors, injected with acridine orange (5 $\mu \mathrm{g} /$ tumor), were captured with exposure times of $2 \mathrm{~min}$, using a fluorescein filter $(470 \mathrm{~nm})$ for excitation and a Cy5 excitation filter $(640 \mathrm{~nm})$ for emission. Red-green-blue (RGB) images of the tumors were pseudocolored for optimal display contrast with custom-designed Biological Detection Systems (BDS, Pittsburgh, PA, USA) imaging software.

\section{Fluorescence Imaging of Tumor Sections}

A Zeiss Axiovert platform-based, modified multimode microscope (9), equipped with a Plan Neofluar $40 X, 1.3$ N.A. oil immersion objective and the cooled CCD camera were used to view and record images of tissue sections prepared from tumors. Acetone-fixed, 5- $\mu \mathrm{m}$ tissue sections, prepared from snap-frozen VGP and MGP melanomas injected with human tyrosinase and RSV promoter-driven human bFGF or FGFR-1 antisense construct and cyanine fluorochrome-labeled antibodies were imaged for $10 \mathrm{sec}$.

Acetone-fixed, adjacent 5- $\mu \mathrm{m}$ tissue sections, obtained from snap-frozen tumors, which had received bFGF or FGFR-1 antisense construct but no cyanine fluorochrome-labeled antibodies were incubated for $60 \mathrm{~min}$ in a $37^{\circ} \mathrm{C}$ humidified chamber with $\mathrm{Cy} 3-$ conjugated anti-human VEGF-D, Cy5-conjugated antihuman bFGF, or Cy7-conjugated anti-human FGFR-1 antibody. Thereafter, the sections were rinsed several times with phosphate-buffered saline (PBS), mounted with xylene-based Cytoseal (Stephens Scientific, Riverdale, NJ, USA), and imaged for $10 \mathrm{sec}$ with the respective cyanine excitation and emission filters.

Acetone-fixed, 5- $\mu \mathrm{m}$ tissue sections, prepared from tumors that had been inoculated with bFGF or FGFR-1 antisense construct but not with cyanineconjugated antibodies or acridine orange, were stained with a rhodamine-based ApopTaq in situ apoptosis detection kit (Intergen, Norcross, GA, USA). The rhodamine-stained tumor sections were imaged for 5 sec, using $\mathrm{Cy} 3$ excitation and emission filters. RGB images of the tumor sections were generated using BDS imaging software.

\section{Results}

Intratumoral Injection of Tyr-bFGF or Tyr-FGFR-1 Antisense Construct Inhibits Melanoma Growth

Previous studies, conducted with VGP and MGP human melanoma cells grown as subcutaneous tumors 
in nude mice, revealed that antisense targeting of bFGF and FGFR-1 blocks intratumoral angiogenesis and tumor growth (4). To inhibit production of bFGF and FGFR-1, the melanomas were injected with Epstein-Barr virus (EBV)-based mammalian expression vectors that expressed antisense-oriented, fulllength cDNA for human bFGF or FGFR-1 under the control of a constitutive RSV promoter, which is expressed in different types of mammalian cells, including epithelial and endothelial cells, as well as fibroblasts.

To obtain a more detailed insight into regulation of melanoma angiogenesis, addressing in particular the issue of potential "cross-talk" between bFGF/ FGFR-1 produced by melanoma cells and angiogenic molecules emanating from endothelial cells lining the melanoma-interspersing vasculature, we replaced the RSV promoter in the bFGF and FGFR1 antisense-oriented plasmid with a 430-bp cDNA fragment of the human tyrosinase promoter (8), which is strongly expressed in cells of the human melanocytic lineage. To assess the efficacy of the TyrbFGF and Tyr-FGFR-1 antisense constructs in vitro, we transfected VGP and MGP melanoma cell lines with the respective plasmids. As shown previously in the case of VGP and MGP melanoma cells transfected with the RSV-bFGF and RSV-FGFR-1 constructs (4), transfection of the Tyr-bFGF and Tyr-FGFR-1 antisense-oriented plasmids did not yield hygromycin-resistant colonies because the melanoma cells died within 2 days following transfection (data not shown).

To test the impact of intratumoral injections of the Tyr-bFGF and Tyr-FGFR-1 antisense constructs on melanoma growth in vivo, VGP and MGP melanoma cells, grown in nude mice as subcutaneous tumors to a diameter of $5 \mathrm{~mm}$, were injected with an initial dose of $100 \mu \mathrm{g}$ of plasmid DNA mixed with $10 \mathrm{nmol}$ of DC-Chol liposomes, followed by twice-weekly intratumoral injections of $20 \mu \mathrm{g}$ of plasmid DNA mixed with $10 \mathrm{nmol}$ of DCChol liposomes. Figure 1 shows the results of targeting studies in MGP melanomas, which were very similar to those obtained upon intratumoral injections of the vector constructs into VGP melanomas (data not shown). Compared to uninjected MGP melanomas and MGP melanomas that received injections with pREP7 vector not containing bFGF or FGFR-1 cDNA, tumors injected with Tyr-bFGF (a) or Tyr-FGFR-1 antisense construct (b) did not grow significantly beyond their initial size. Because these findings provided a first indication that targeting bFGF and FGFR-1 in only the melanoma cells may be as effective in blocking tumor growth as inhibiting expression of bFGF or FGFR-1 in melanoma cells and the melanoma cell-interspersing vasculature (4), we initiated a series of optical imaging studies to visualize and record the biological events that unfold inside melanomas as the result of intratumoral injections of the human tyrosinase versus
RSV promoter-driven bFGF and FGFR-1 antisenseoriented vectors.

Noninvasive In Vivo and Ex Vivo Fluorescence Imaging of bFGF and FGFR-1-Antisense-Targeted Melanomas Reveals Extensive Melanoma Cell Death

Recent advances in real-time, spatiotemporal optical imaging have opened new avenues for intravital documentation and assessment of physiologic functions in malignant and nonmalignant diseases. Using fluorochrome-based imaging, we captured over a period of 2 weeks, live images of VGP and MGP melanomas growing as subcutaneous tumors in nude mice. Control tumors were comprised of uninjected melanomas, melanomas that received only liposomes, and melanomas that were injected with pREP7 vector mixed with liposomes. The groups of experimental tumors received Tyr-bFGF, Tyr-FGFR1, RSV-bFGF, or RSV-FGFR-1 antisense construct mixed with liposomes. On the days following the twice-weekly injections with the respective vector construct or liposomes only, the tumors were inoculated with a Cy7-conjugated S100 antibody, which binds to melanoma cells (10), and a Cy5-labeled antibody to CD31 antigen, which is expressed on endothelial cells (11). Examples of images of MGP melanomas, captured noninvasively, through the skin of the live animals at 2 weeks of intratumoral injections, are presented in Figure 2. At that point, the large tumors representing the three different groups of controls (Fig. 2A) were generating bright fluorescent images upon injection with the Cy7S100 (a-c, pseudocolored green) and Cy5-CD31 antibody (d-f, pseudocolored red). In contrast, the experimental groups of melanomas (Fig. 2B), injected with Tyr-bFGF (a and e), Tyr-FGFR-1 (b and f), RSV-bFGF (c and g), or RSV-FGFR-1 vector ( $\mathrm{d}$ and h), yielded significantly weaker, and in some cases also smaller Cy7-S100 (a-d) and Cy5-CD31 (e-f) fluorescent images.

Because the in vivo images of the bFGF and FGFR-1 antisense-targeted tumors, recorded over a period of 2 weeks, indicated that the melanoma cells and intratumoral blood vessels might be dying, we sacrificed all tumor-bearing animals and imaged tissue sections from the resected tumors. Representative examples of these ex vivo tumor images, acquired in the Cy5 and Cy7 channels and then superimposed, are depicted in Figure 2C. Cy7-S100/ Cy5-CD31 images of an uninjected MGP melanoma (a), an MGP melanoma that received only liposomes (b), and an MGP melanoma injected with pREP7 vector/liposomes (c) showed a dense population of melanoma cells (pseudocolored green), interspersed by large blood vessels (pseudocolored red). In contrast, ex vivo images of Tyr-bFGF (d), Tyr-FGFR-1 (e), RSV-bFGF (f), or RSV-FGFR- 1 antisense-targeted melanomas (g) revealed considerably fewer Cy7-S100 fluorescent melanoma cells. 

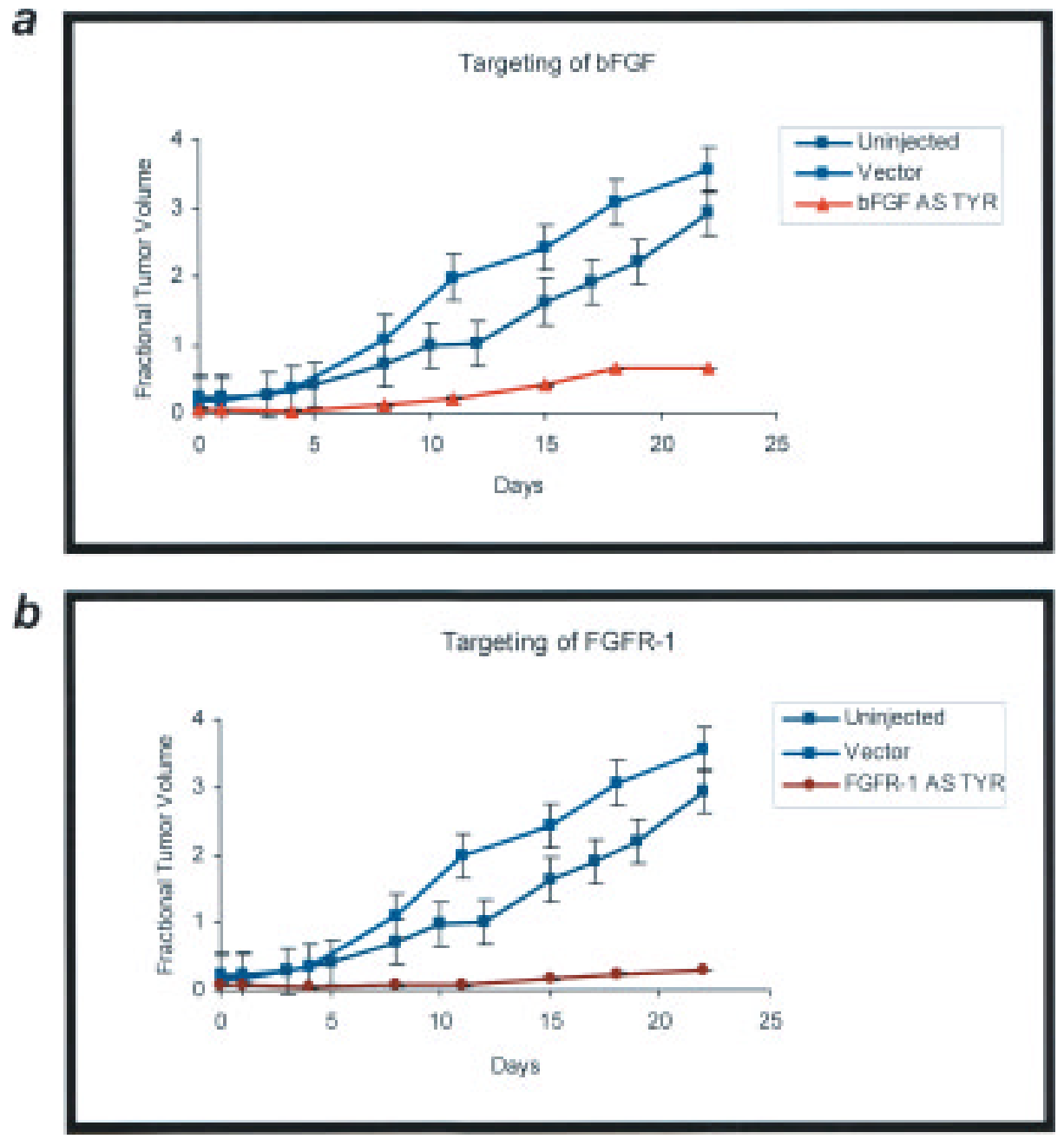

Fig. 1. Tumor volume of human melanomas, inoculated with Tyr-bFGF or Tyr-FGFR-1 antisense construct-liposome complexes. Nude mice were injected subcutaneously on their dorsal site with $5 \times 10^{6} \mathrm{MGP}$ melanoma cells/animal. When the tumors had reached $5 \mathrm{~mm}$ in perpendicular diameter, they were inoculated with $100 \mu \mathrm{g}$ of plasmid DNA, mixed with $10 \mathrm{nmol}$ of DC-Chol liposomes, followed by twice weekly injections of $20 \mu \mathrm{g}$ of plasmid DNA mixed with $10 \mathrm{nmol}$ of DC-Chol liposomes. Perpendicular tumor diameters were measured daily. All animals were sacrificed on day 22 when the mice bearing control tumors reached the maximal allowable size of $2 \mathrm{~cm}$ in perpendicular diameter. (a) Volume of MGP melanomas injected with Tyr-bFGF antisense construct. (b) Volume of MGP melanomas that received Tyr-FGFR-1 antisense construct. Volumes of control tumors, which were not inoculated or had received pREP7 vector construct, not containing human bFGF or FGFR-1 antisense-oriented cDNA, are the same in both panels. Means are depicted, and standard error bars are shown for each group of tumors.

\section{Tyr-bFGF/FGFR-1-Targeted Melanomas Do Not Activate Expression of Another Growth Factor to Override the Blocked Melanoma Cell Growth}

To investigate whether the melanoma cells, upon blocking their production of bFGF or FGFR-1 with the Tyr-bFGF or Tyr-FGFR-1 antisense construct, activated or increased expression of another molecule such as vascular endothelial growth factor (VEGF) in an effort to circumvent or override the arrest in proliferation, we stained tissue sections from the Tyr-bFGF and Tyr-FGFR-1 antisense-targeted tumors with a VEGF-D antibody conjugated to Cy3. Uninjected melanomas, melanomas injected with pREP7 construct (data not shown), and melanomas that received only DC-Chol liposomes (Fig. 3a, pseudocolored red), exhibited strong expression of VEGF in the tumor vasculature and to a lesser extent in the melanoma cells. In contrast, tissue sections of the Tyr-bFGF (Fig. 3b) and Tyr-FGFR-1 antisensetargeted melanomas (Fig. 3c) showed no VEGF staining, regardless of whether the tissue sections were derived from the center or periphery of the tumors. In addition, we probed tissue sections from the same tumors with a Cy5-conjugated bFGF and Cy7-conjugated FGFR-1 antibody. Sections prepared from control tumors showed prominent bFGF (Fig. 3d, pseudocolored yellow) and FGFR-1 staining (Fig. 3g, pseudocolored green) of the melanoma cells, whereas tissue sections from Tyr-bFGF (Figs 3e and $3 \mathrm{~h}$ ) and Tyr-FGFR-1 (Figs $3 \mathrm{f}$ and $3 \mathrm{i}$ ) antisense- 
targeted tumors revealed only a few melanoma cells along their periphery, which demonstrates the presence of bFGF (Figs $3 \mathrm{e}$ and $3 \mathrm{f}$ ) or FGFR-1 protein (Figs $3 \mathrm{~h}$ and $3 \mathrm{i}$ ).

\section{Blocking Production of bFGF or FGFR-1 in Melanoma Cells Induces Massive and Irreversible Apoptosis}

To address the possibility that blocking expression of bFGF and likewise, FGFR-1 in the melanoma cells did lead to extensive melanoma cell apoptosis, Tyr-bFGF and Tyr-FGFR-1 antisense-targeted melanomas were injected with the fluorescent dye acridine orange, which binds to fragmented doublestranded DNA and thus serves as a marker for apoptotic cells (12). Noninvasive, live fluorescence imaging of the Tyr-bFGF and Tyr-FGFR-1 antisensetargeted tumors started to reveal the presence of small clusters of acridine orange-labeled melanoma a
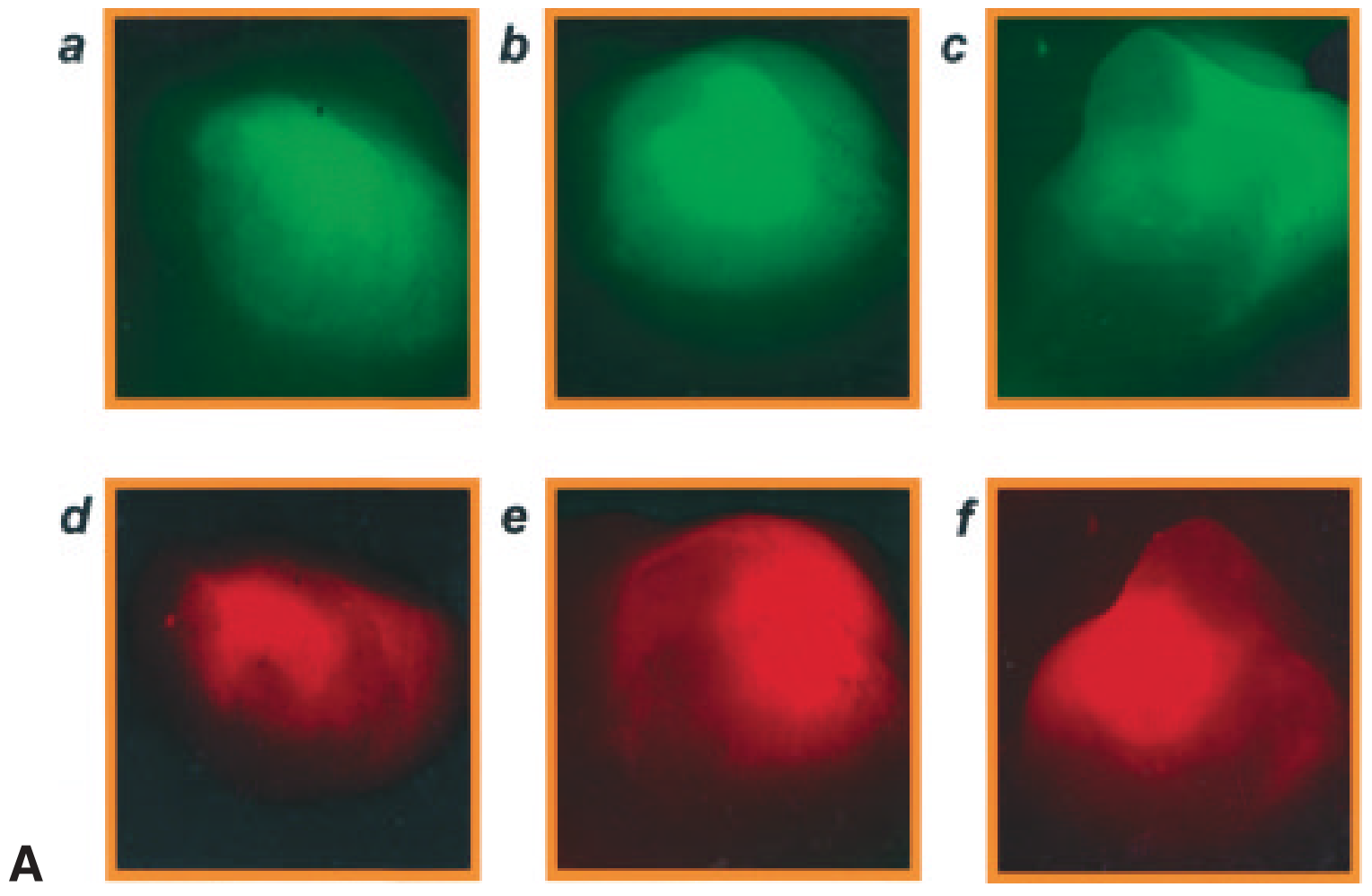

Fig. 2. In vivo and ex vivo fluorescent images of melanomas injected with tyrosinase or RSV promoter-driven bFGF or FGFR-1 antisense constructs. MGP melanomas, grown as subcutaneous tumors on the dorsal site of nude mice, were injected with tyrosinase or RSV promoter-driven bFGF or FGFR-1 antisense constructs as described in Figure 1. Twenty-four hours following the second injection of plasmid construct, each tumor was injected with $5 \mu \mathrm{g}$ of Cy5-conjugated CD 31 antibody. Two hours later, the tumors were imaged noninvasively with a Cy5 excitation and emission filter. Thereafter, the tumors were injected with $5 \mu \mathrm{g}$ of Cy7conjugated S100 antibody, and 2 hours later, the tumors were imaged noninvasively using a Cy7 emission and excitation filter. The three groups of control tumors were MGP melanomas, which received fluorochrome-conjugated S100 and CD31 antibody but no plasmid, MGP melanomas that received DC-Chol liposomes and the two fluorochrome-conjugated antibodies, and tumors that were injected with pREP7 vector and the two cyanine dye-conjugated antibodies. Noninvasive, dynamic fluorescence imaging of the tumors was performed twice a week. All animals were sacrificed when the control tumor-bearing mice reached the maximal allowable size of $2 \mathrm{~cm}$ in perpendicular diameter. Examples of pseudocolored, noninvasive fluorescent images of control tumors, captured after 2 weeks of intratumoral antibody injections, are depicted in (A). (Fig. 2A, panels a and d) An MGP melanoma, which received only Cy7conjugated S100 (panel a, pseudocolored red), and Cy5-conjuagted CD31 antibody (panel d, pseudocolored green). Panels (b) and (e) show an MGP melanoma injected with DC-Chol liposomes and Cy7-S100 (panel b) and Cy5-CD31 antibody (panel e). (Fig. 2A, panels $\mathrm{c}$ and f) A tumor that was inoculated with pREP7 vector, mixed with DC-Chol liposomes, followed by injection of Cy7-S100 (panel c) and Cy7-CD31 antibody (panel f). (Fig. 2B, panels a-h) Pseudocolored, noninvasively captured fluorescent images of MGP melanomas 2 weeks after injections with tyrosinase or RSV-promoter-driven bFGF or FGFR-1 antisense constructs and fluorochromeconjugated S100 and CD31 antibody. A tumor that received Tyr-bFGF antisense construct and Cy7-S100 antibody is displayed in (panel a). A Cy5-CD31 fluorescent image of the same tumor is shown in (panel e). The tumor shown in panels $b$ and $\mathbf{f}$ received Tyr-FGFR-1 antisense construct and Cy7-S100 (panel b) and Cy5-CD31 antibody (panel f). Panel c depicts a tumor inoculated with RSV-bFGF antisense construct and CY7-S100 and Cy5-CD31 antibody (panel g). An MGP melanoma, which received RSV-FGFR-1 antisense-oriented plasmid and Cy7-S100 and Cy5-CD31 antibody, is presented in panels d and h, respectively. Displayed in Fig. 2C, panels a-g are superimposed, pseudocolored fluorescent images of tissue sections, which were prepared from the noninvasively imaged tumors, depicted in Fig. 2A, and imaged in the Cy5 and Cy7 channel. (Panel a) uninjected MGP melanoma; (panel b) MGP melanoma injected with DC-Chol liposomes; (panel c) MGP melanoma inoculated with pREP7 vector; (panel d) tumor inoculated with Tyr-bFGF antisense construct; (panel e) tumor injected with Tyr-FGFR-1 antisense construct; (panel f) MGP melanoma that received RSV-bFGF antisense-oriented vector; and (panel g) tumor injected with RSV-FGFR-1 antisense construct. Melanoma cells are pseudocolored, green, and tumor-interspersing blood vessels, red. 

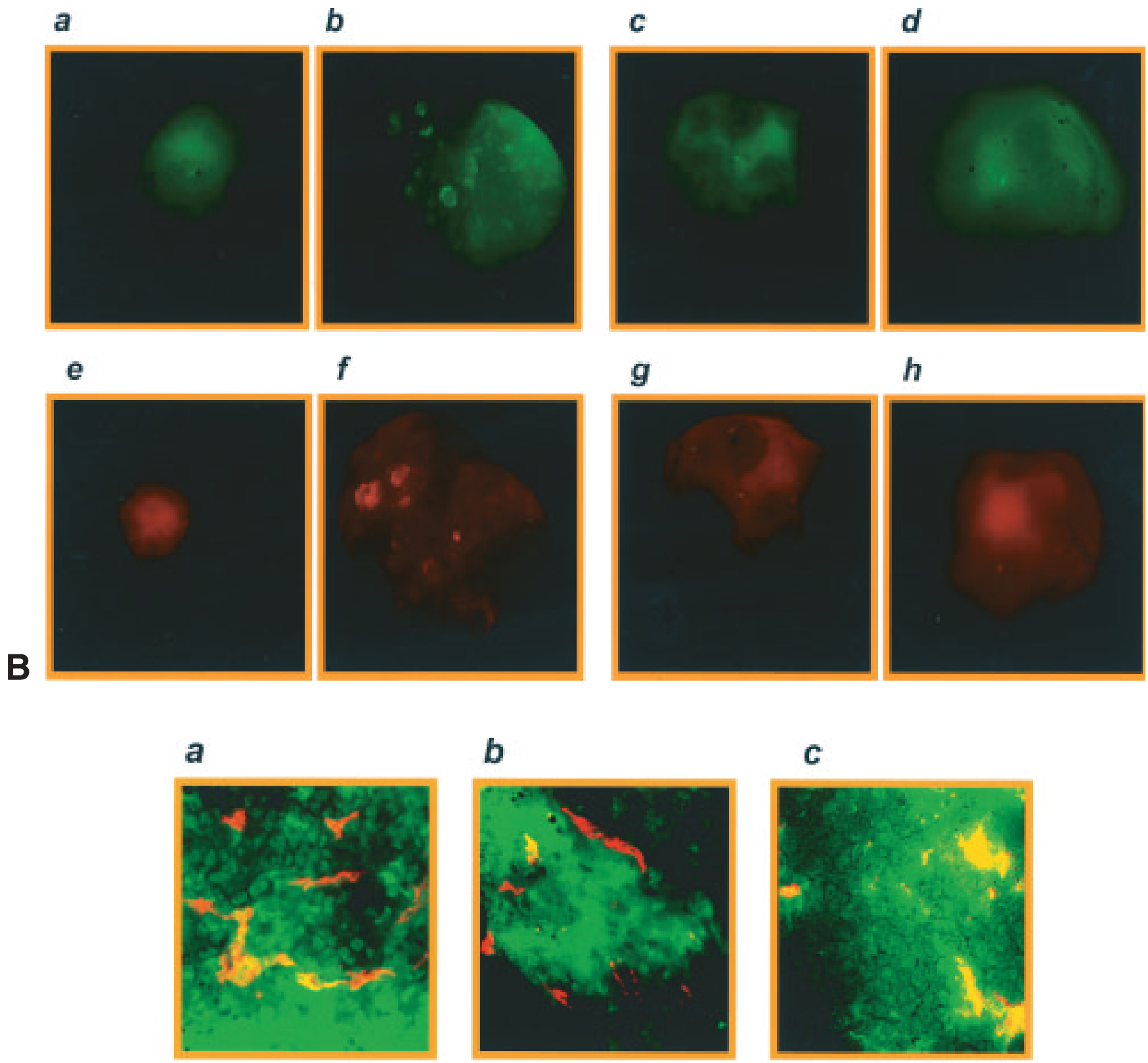

\section{b}
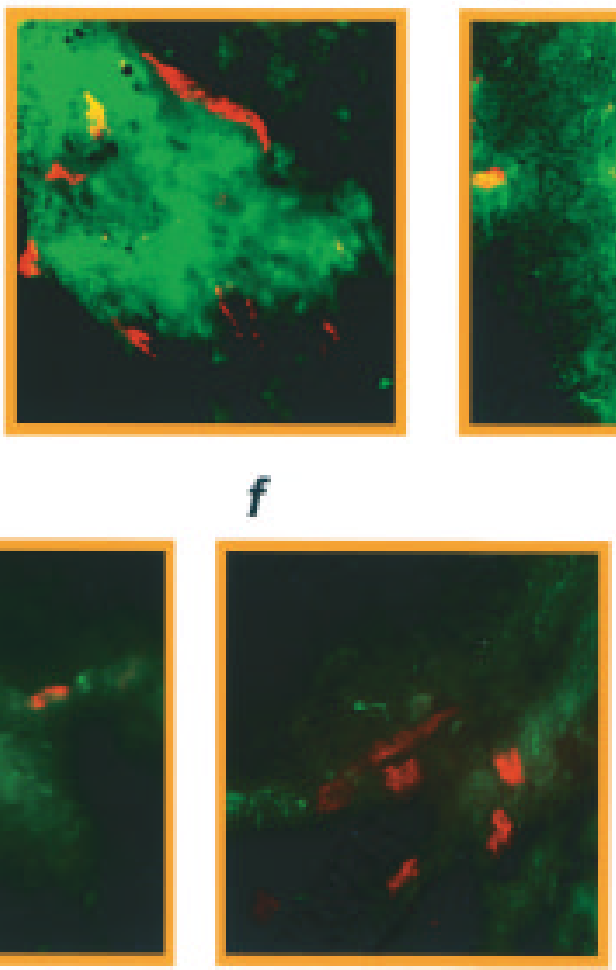

c

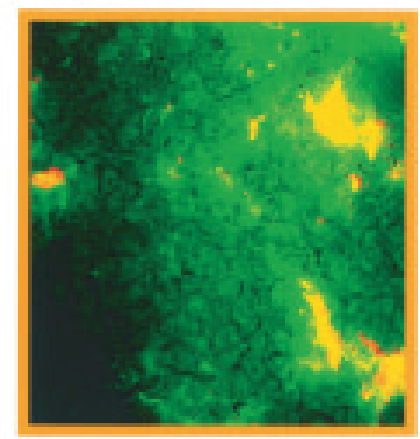

g

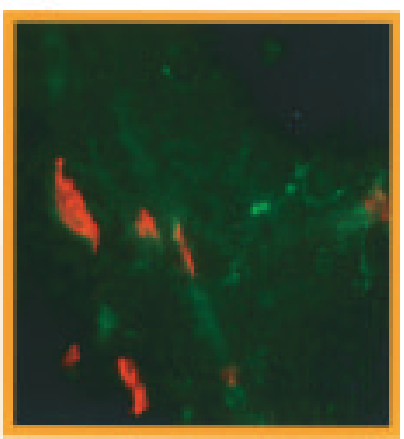

Fig. 2. Continued 
a

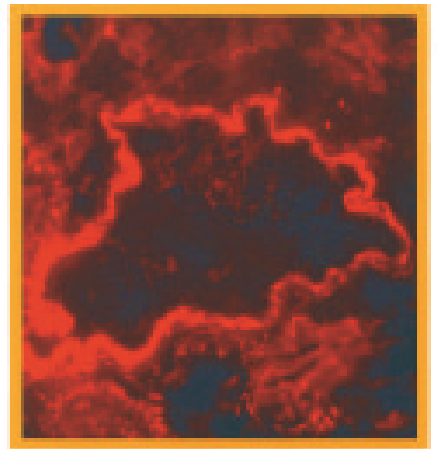

Q

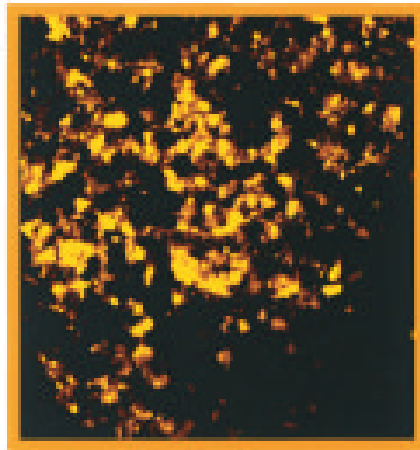

g

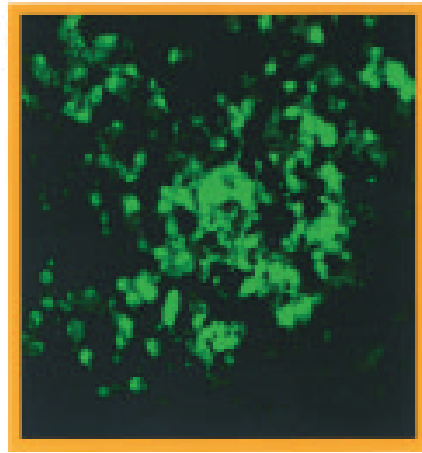

b

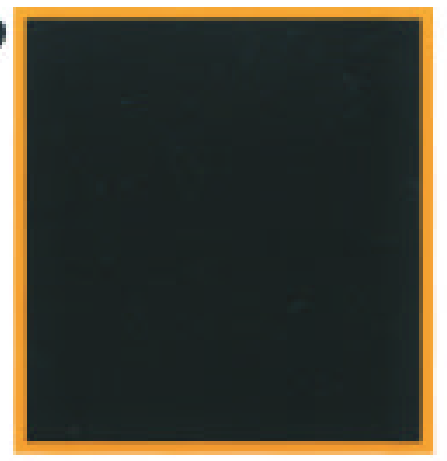

e

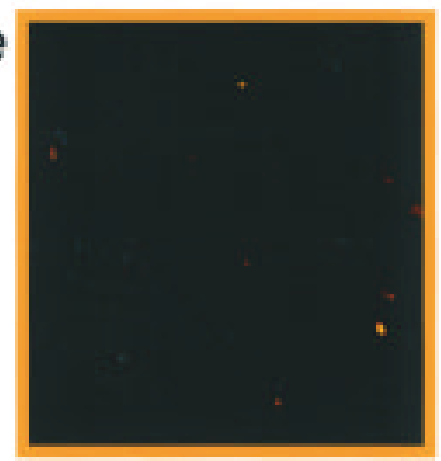

h

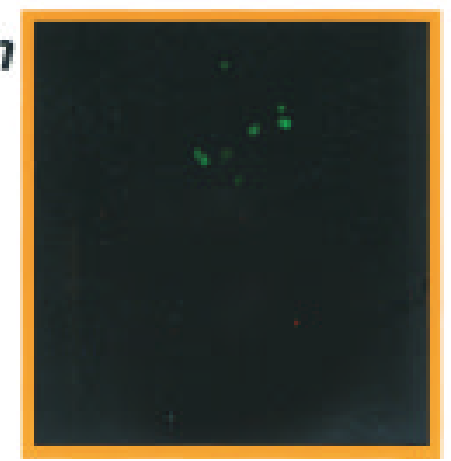

C

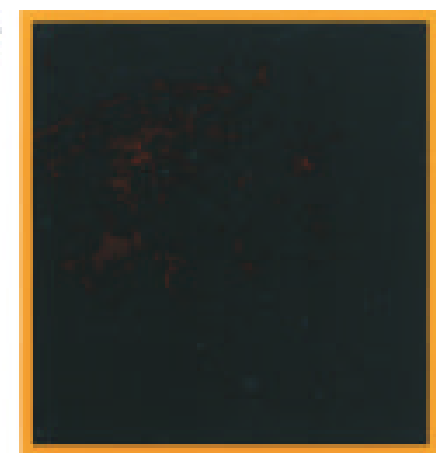

$f$

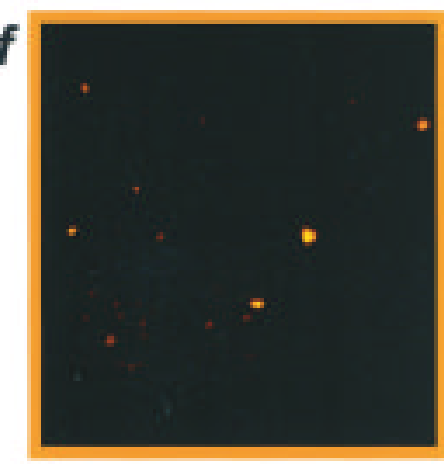

i

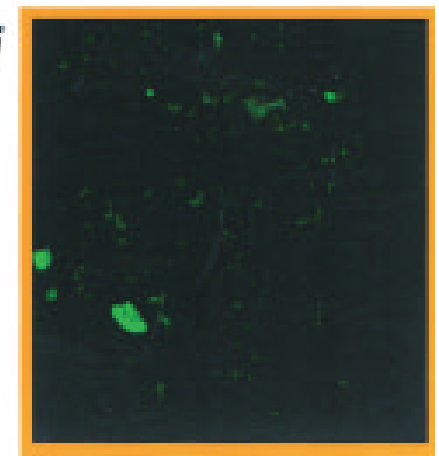

Fig. 3. Images of VEGF, bFGF, and FGFR-1-stained tissue sections of melanomas injected with Tyr-bFGF or Tyr-FGFR-1 antisense construct. Adjacent tissue sections, prepared from control tumors and tumors that been injected twice weekly for a period of 4 weeks with Tyr-bFGF or Tyr-FGFR-1 antisense construct, were probed with Cy3-conjugated VEGF-D, Cy5-conjugated bFGF, and Cy7-conjugated FGFR-1 antibody. Depicted in panels a, $\mathbf{d}$, and $\mathbf{g}$ are tissue sections, obtained from the center of an MGP melanoma, injected with DC-Chol liposomes. Panel a shows staining for VEGF-D (pseudocolored red), d for bFGF (pseudocolored yellow), and $\mathbf{g}$ for FGFR-1 (pseudocolored green). Displayed in panels $\mathbf{b}$, $\mathbf{e}$, and $\mathbf{h}$ are tissue sections of an MGP melanoma that had been inoculated with the Tyr-bFGF antisense-oriented plasmid. Panel b depicts a tissue section, stained with Cy3-VEGF-D antibody, e a tissue section probed with Cy5-bFGF antibody, and $\mathbf{h}$ a tumor section probed with Cy7-FGFR-1 antibody. Images of stained sections, obtained from an MGP melanoma injected with Tyr-FGFR-1 antisense construct, are depicted in panels c, f, and i. The melanoma section shown in panel $\mathbf{c}$ was probed with Cy3-VEGF-D antibody, the tissue section, shown in panel f, with Cy5-bFGF antibody, and the melanoma section presented in panel $\mathbf{i}$, with Cy7-FGFR-1 antibody.

cells within 24-48 hrs following the first intratumoral injection of either plasmid construct. Over a period of 2 weeks, as the tumors received further injections of the plasmid/liposome complexes, the acridine orange-positive melanoma cell clusters became very prominent throughout the tumors. In contrast, acridine orange injections of the three different groups of control tumors yielded barely detectable acridine orange-based fluorescence in the images. Figure $4 \mathrm{a}$ is a representative in vivo image of a control tumor that was inoculated twice a week for 2 weeks with DC-Chol liposomes, followed by a single injection of acridine orange. Figures $4 \mathrm{~b}$ and $4 \mathrm{c}$ are noninvasively captured acridine orange images of a Tyr-bFGF (Fig. 4b) and a Tyr-FGFR-1 (Fig. 4c) antisense-targeted melanoma, which had received twice-weekly injections of the vector constructs for a period of 2 weeks.

To independently verify the observation that melanomas are driven into apoptosis as the result of blocking bFGF and FGFR-1 production in the melanoma cells, tissue sections prepared from VGP and MGP melanomas, which had been inoculated twice a week for 4 weeks with the Tyr-bFGF or 

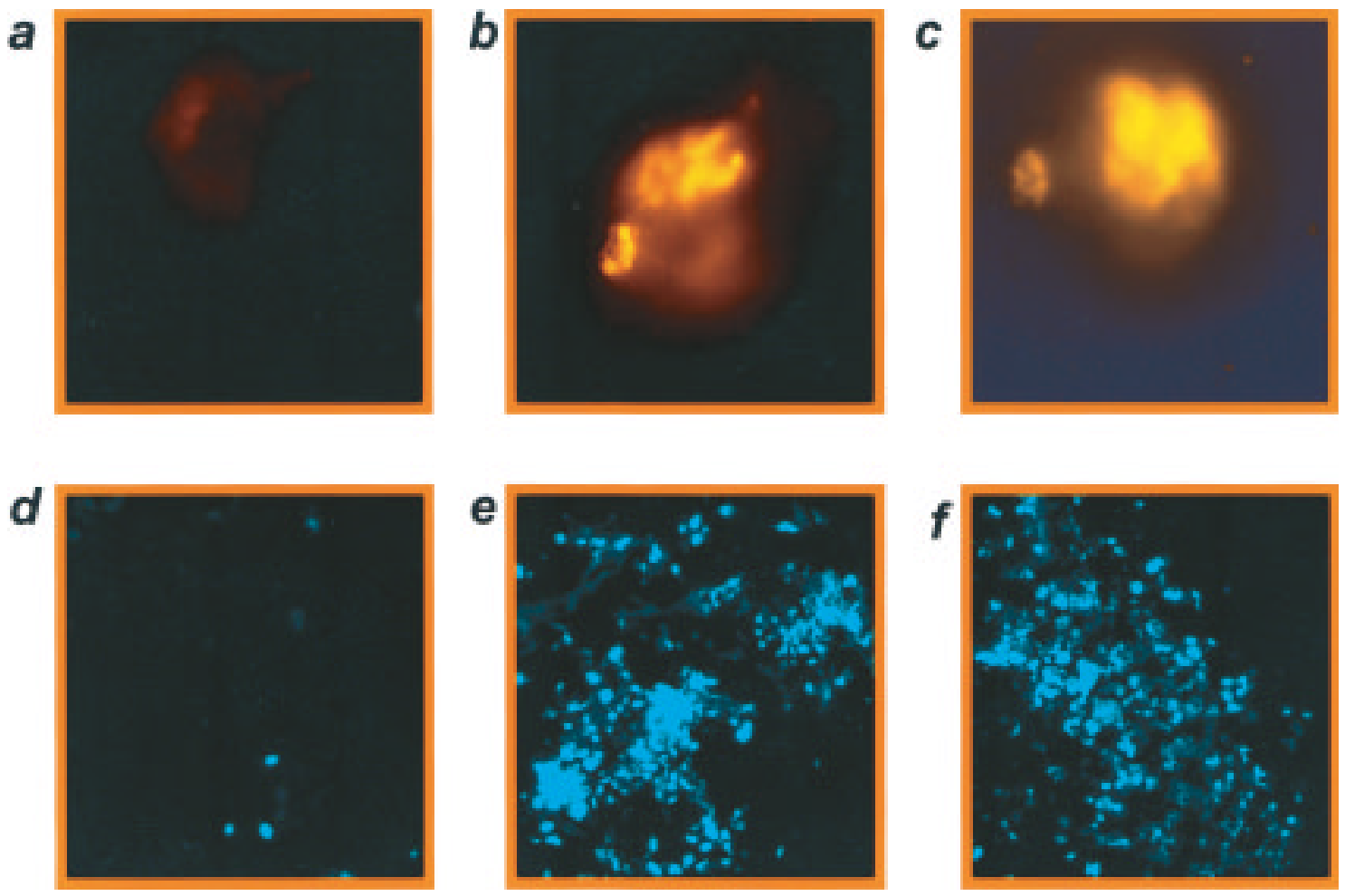

Fig. 4. In vivo and in vitro images of Tyr-bFGF and Tyr-FGFR-1-targeted melanomas demonstrate melanoma cell apoptosis. MGP melanomas, inoculated twice a week for 2 weeks with Tyr-bFGF or Tyr-FGFR-1 antisense construct and Cy7-S100 and Cy5CD31 antibody and, similarly, control tumors that received only Cy7-S100 and Cy5-CD31 antibody, or injections with DC-Chol liposomes or pREP7 vector and the two fluorochrome-conjugated antibodies, were injected with acridine orange. Two hours later, the tumors were imaged noninvasively in the Cy3 channel. (a) Image of an acridine orange-injected MGP melanoma, injected with DC-Chol liposomes. (b) Image of an acridine orange-inoculated Tyr-bFGF-targeted MGP melanoma.(c) Image of an acridine orangeinjected Tyr-FGFR-1-targeted MGP melanoma. Tissue sections, prepared from MGP melanomas that had been injected twice a week for 4 weeks with Tyr-bFGF or Tyr-FGFR-1 antisense construct, were subjected to rhodamine-based TUNEL staining. (d) Image of a tissue section of an MGP melanoma, inoculated with DC-Chol liposomes. (e) A rhodamine-stained image of a tissue section, prepared from a Tyr-bFGF antisense-targeted MGP melanoma. (f) A rhodamine image of a section obtained from a Tyr-FGFR-1 plasmid-injected MGP melanoma. Images of acridine orange-injected tumors were pseudocolored orange, and rhodamine images of melanoma sections were assigned the pseudocolor blue.

Tyr-FGFR-1 construct, were subjected to rhodaminebased TUNEL staining (13). Like in the case of the noninvasively captured in vivo images, the ex vivo images of the Tyr-bFGF and Tyr-FGFR-1-targeted melanomas (Figs 4e and 4f) demonstrated extensive melanoma cell apoptosis, whereas the control tumors did not (Fig. 4d).

Despite the fact that the Tyr-bFGF and TyrFGFR-1 antisense-targeted melanoma cells revealed an extensive degree of apoptosis, there nevertheless was a possibility that some of the melanoma cells remained viable and over time, would be able to repopulate the tumors. To address this possibility, MGP melanomas were injected twice weekly with the Tyr-bFGF or Tyr-FGFR-1 plasmid-liposome complexes. On day 25 following the first intratumoral injection, the inoculations were stopped and thereupon, tumor sizes were measured on a daily basis for another 15 days. As depicted in Figure 5, we did not obtain experimental evidence that the Tyr-bFGF or Tyr-FGFR-1 antisense-targeted melanomas were able to overcome their growth arrest. Furthermore, fluorescence imaging of tissue sections prepared from the tumors, resected on day 40, did not reveal the presence of Cy7-S100labeled melanoma cells or Cy5-CD31-stained blood vessels (data not shown).

\section{Discussion}

Gaining insights into the molecular and cellular mechanisms governing tumor angiogenesis has become one of the central themes in cancer biology (14-16), and the characterization of pro- and anti-angiogenic molecules has led to a series of clinical trials to assess whether anti-angiogenic treatment can be an effective strategy to restrict tumor growth $(15,17,18)$.

In line with these topics, the first series of studies, conducted more than a decade ago, demonstrated that the pro-angiogenic molecule, bFGF, and one of its receptors, FGFR-1, are produced at high levels by VGP and MGP melanoma cells and govern their proliferation (1-3). Subsequent investigations, conducted in vivo, revealed that blocking expression of 


\section{Targeting of bFGF and FGFR-1}

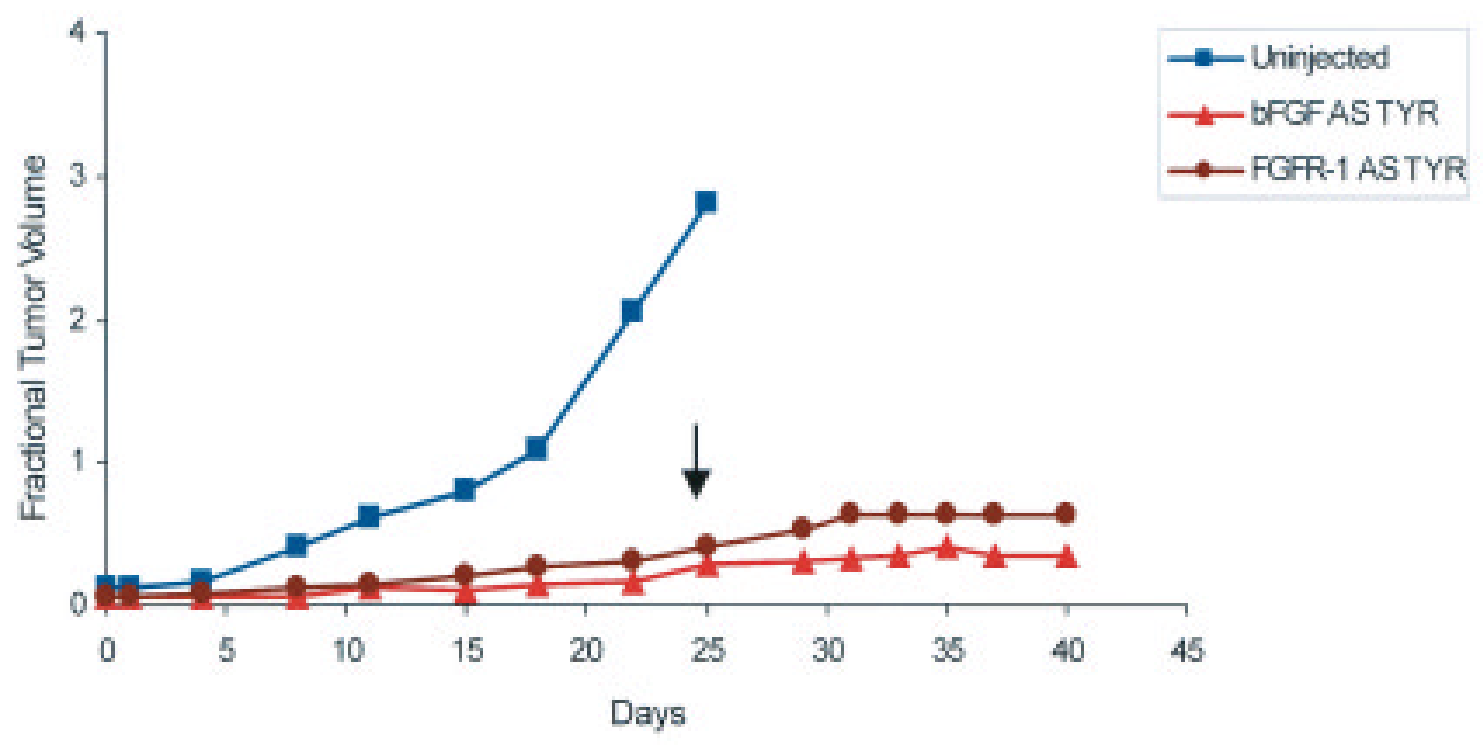

Fig. 5. Tumor volume of melanomas upon cessation of Tyr-bFGF and Tyr-FGFR-1 plasmid injections. MGP melanomas were inoculated twice weekly with Tyr-bFGF or Tyr-FGFR-1 antisense construct-liposome complexes. On day 25, following the first injection of either vector construct, the injections were stopped and thereafter, tumor sizes were measured daily until day 40, when the animals were sacrificed. The arrow indicates the final day of intratumoral injections. Animals bearing uninjected control tumors were sacrificed on day 25 since their tumors had reached the maximal allowable size. Standard errors (not shown) were 0.26 for Tyr-bFGF and 0.14 for Tyr-FGFR-1 antisense construct-injected tumors.

bFGF or FGFR-1 in VGP and MGP melanomas, grown as subcutaneous tumors in nude mice, causes tumor growth arrest as a result of blocked intratumoral angiogenesis (4). However, these previous in vivo studies involved intratumoral injections of bFGF and FGFR-1 antisense constructs under the control of a constitutive RSV promoter, which is expressed in different cell types, including endothelial cells. Thus, it was not possible to determine whether the observed tumor growth arrest and blocked intratumoral angiogenesis were the result of blocked production of bFGF/FGFR-1 in the melanoma cells or in the melanoma cells and the intratumoral vasculature.

The answer to this question is now provided by the present in vivo study, which demonstrates that inhibiting expression of bFGF and likewise, FGFR-1, in only the melanoma cells is as effective in blocking tumor growth as simultaneously inhibiting bFGF/FGFR-1 synthesis in the melanoma cells and the melanoma cell-interspersing blood vessels. Interestingly, albeit surprisingly, the experiments also revealed that blocking expression of bFGF or FGFR1 in the melanoma cells did not cause the melanoma cells to activate or increase production of another angiogenic molecule such as VEGF or another nonangiogenic growth factor/growth factor receptor to sustain their proliferation. Similarly, another "rescue mechanism" one may have predicted to be switched on, at least in the Tyr-bFGF antisensetargeted melanomas, was for bFGF, produced by the endothelial cells lining the intratumoral blood vessels, to bind to FGFR-1 on the melanoma cells and thereby ensure continuous growth of the tumors. However, neither of these potential salvage pathways appeared to be in place. Instead, within a day or two after the tumors had received the first dose of Tyr-bFGF or Tyr-FGFR-1 antisense construct and while the melanoma cells were still interspersed by a large number of visibly viable blood vessels, noninvasively captured live images revealed that clusters of melanoma cells started to exhibit the first signs of apoptosis. In fact, following continuous intratumoral injection of each plasmid construct, it was the melanoma cells rather than the intratumoral blood vessels that underwent massive apoptosis.

Although the model explored in this study provides a detailed picture of the biological events set in motion by the specific targeting of bFGF and FGFR-1 
in VGP and MGP human melanoma cells, the results, in turn, raise two important questions. First, how representative will these findings be with respect to other solid malignancies that, like melanoma, express high levels of pro-angiogenic molecules, which are also produced by the tumor vasculature? The second question is what happens to the biology of advanced stage melanomas upon blocking expression of bFGF and FGFR-1 exclusively in the melanoma cell-interspersing vasculature. We are in the process of experimentally addressing this latter issue. In addition to having provided important new insights into the function of bFGF and FGFR-1 in advanced stage human melanomas, which may guide potential new melanoma therapy and angiogenesis-based investigations into a different direction, our study also illustrates the ability of high-resolution, cyanine dye-based fluorescent imaging to visualize and track, through the skin of live animals, the results of intratumorally targeting genes in a human neoplasm growing as subcutaneous xenografts.

\section{Acknowledgments}

We would like to thank C. Goding for providing a human tyrosinase promoter-containing plasmid, L. Huang for providing DC-Chol liposomes, S. Mujumdar for activation of Cy7, and J. Montibeller for initial help with Cy7 conjugation of antibody. This work was supported by a grant from the National Science Foundation (D.L.F.) and the National Institutes of Health (D.B.).

\section{References}

1. Halaban R, Kwon BS, Ghosh S, Delli-Bovi P, Baird A. (1988) bFGF as an autocrine growth factor for human melanomas. Oncogene Res. 3: 177-186.

2. Becker D, Meier CB, Herlyn M. (1989) Proliferation of human malignant melanomas is inhibited by antisense oligodeoxynucleotides targeted against basic fibroblast growth factor. EMBO J. 8: 3685-3691.

3. Becker D, Lee PL, Rodeck U, Herlyn M. (1992) Inhibition of the fibroblast growth factor receptor 1 (FGFR-1) gene in human melanocytes and malignant melanomas leads to inhibition of proliferation and signs indicative of differentiation Oncogene 7: 2302-2313.

4. Wang Y, Becker D. (1997) Antisense targeting of bFGF/ FGFR-1 in human melanomas inhibits intratumoral angiogenesis and tumor growth. Nat. Med. 3: 887-893.

5. Mujumdar RB, Ernst LA, Mujumdar SR, Lewis CJ, Waggoner AS. (1993) Cyanine labeling reagents: Sulfoindocyanine succinimidyl esters. Bioconjugate Chem. 4: 105-108.

6. Ballou B, Fisher GW, Farkas DL, Hakala TR. (1997) Tumor detection and visualization using cyanine fluorochromelabeled antibodies. Biotechnol. Prog. 13: 649-658.

7. Farkas DL, Du C, Fisher GW, et al. (1998) Non-invasive image acquisition and advanced processing in optical bioimaging. Comput. Med. Imaging Graph. 22: 89-102.

8. Bentley NJ, Eisen T, Goding CR. (1994) Melanocyte-specific expression of the human tyrosinase promoter: activation by the microphthalmia gene product and role of the initiator. Mol. Cell Biol. 14: 7996-8006.

9. Farkas DL, Baxter G, DeBiasio RL, et al. (1993) Multimode light microscopy and the dynamics of molecules, cells, and tissues. Annu. Rev. Physiol. 55: 785-817.

10. Gaynor R, Irie R, Morton D, Herschman H. (1980) S100 protein is present in cultured human malignant melanomas. Nature 286: 400-401.

11. Nickoloff RJ. (1993) PECAM-1 (CD31) is expressed on proliferating endothelial cells, stromal spindle-shaped cells, and dermal dendrocytes in Kaposi's sarcoma. J. Clin. Pathol. 43: $752-757$.

12. Darzynkiewicz Z. (1990) Differential staining of DNA and RNA in intact cells and isolated cell nuclei with acridine orange. In Darzynkiewicz Z, Crissman HA (eds). Methods in Cell Biology: Volume 33, Flow Cytometry. New York: Academic Press; 285-298.

13. Gavrieli Y, Sherman Y, Ben-Sasson SA(1992) . Identification of programmed cell death in situ via specific labeling of nuclear DNA fragmentation. J. Cell Biol. 19: 493-501.

14. Carmeliet P, Jain RK. (2000) Angiogenesis in cancer and other diseases. Nature 407: 249-257.

15. Kerbel RS. (2000) Tumor angiogenesis: past, present and the near future. Carcinogenesis 21: 505-515.

16. Ramanujan S, Koenig GC, Padera TP, Stoll BR, Jain RK. (2000) Local imbalance of proangiogenic and antiangiogenic factors: A potential mechanism of focal necrosis and dormancy in tumors. Cancer Res. 60: 1442-1448.

17. Ferrara N, Alitalo K. (1999) Clinical applications of angiogenic growth factors and their inhibitors. Nat. Med. 12: 1359-1364.

18. Kerbel RS. (2001) Clinical trials of antiangiogenic drugs: Opportunities, problems, and assessment of initial results. J. Clin. Oncol. 19: 45-51. 